\title{
Spontaneous polarization in the deuterated and undeuterated proton glass $\mathrm{Rb} 1-$ $\mathrm{x}(\mathrm{NH} 4) \mathrm{xH} 2 \mathrm{AsO} 4$
}

\section{Authors: N.J. Pinto and V. Hugo Schmidt}

This is an Accepted Manuscript of an article published in Ferroelectrics on [date of publication], available online: http://www.tandfonline.com/10.1080/00150199308223448.

N.J. Pinto and V.H. Schmidt, "Spontaneous polarization in the deuterated and undeuterated proton glass Rb1-x(NH4)xH2AsO4," Ferroelectrics 141, 207-213 (1993). http://dx.doi.org/10.1080/00150199308223448 


\title{
SPONTANEOUS POLARIZATION IN THE DEUTERATED AND UNDEUTERATED PROTON GLASS $\mathrm{Rb}_{1-x}\left(\mathrm{NH}_{4}\right)_{x} \mathrm{H}_{2} \mathrm{AsO}_{4}$
}

\author{
NICHOLAS J. PINTO and V. HUGO SCHMIDT \\ Department of Physics, Montana State University, \\ Bozeman, Montana 59717 USA
}

(Received August 28, 1992, in final form December 17, 1992)

\begin{abstract}
Spontaneous polarization of mixed single crystals of Rubidium Ammonium Dihydrogen Arsenate (RADA) and its deuterated counterpart (DRADA) are presented together with the pure ferroelectric Rubidium Dihydrogen Arsenate (RDA) and its deuterated counterpart (DRDA). There is a sharp rise in the spontaneous polarization at the ferroelectric transition temperature $T$, for the pure crystals as this transition is first order. The change in the spontaneous polarization in the mixed $(x=0.08)$ crystals below $T$, is gradual however, indicating the presence of acid hydrogens and ammonium cations that are still mobile and in the paraclectric phase. These mixed crystals show coexistence of paraelectric/proton glass and ferroelectric order below the glass "transition" temperature $T_{\sharp}$. The maximum values of the spontaneous polarization in the deuterated samples are greater than in the undeuterated samples. Also, the maximum values of the spontaneous polarization in mixed crystals below 7 , at the lowest temperatures reported is found to be slightly lower than the corresponding values in the pure crystals.
\end{abstract}

Keywords: ferroelectric, spontaneous polarization, proton glass

Proton glass behavior was discovered by Courtens ${ }^{1}$ in 1982 in a mixture of $\mathrm{RbH}_{2} \mathrm{PO}_{4}$ (RDP) and $\mathrm{NH}_{4} \mathrm{H}_{2} \mathrm{PO}_{4}$ (ADP). Here RDP is a pure ferroelectric and ADP is at pure antiferroelectric. In a mixture of the form (RDP) $)_{1-x}(\mathrm{ADP})_{x}$, (RADP), for a certain range of $x$ values, proton glass behavior appears below the "freezing" temperature $T_{g}$ due to frustration between ferroelectric and antiferroelectric ordering. In this range the system makes a smooth transition to the glass regime from the paraelectric regime as temperature is reduced below the glass "transition" temperature. If a spontaneous polarization were present in these mixed crystals it could be measured by conventional means like a Sawyer-Tower ${ }^{2}$ circuit, and its value would depend on the amount of ferroelectric material present. For pure proton glasses the equilibrium spontaneous polarization is zero. In such crystals a quasi-equilibrium polarization can be generated by field-cooling ${ }^{3+}$ the sample down into the nonergodic region.

The crystal structure of these mixed systems is of the $\mathrm{KH}_{2} \mathrm{PO}_{4}$ (KDP) type. A variety of mixed systems of the KDP type can be prepared by substituting $\mathrm{Rb}$ for $\mathrm{K}$ and $\mathrm{As}$ for $\mathrm{P}$. In addition, these systems can be deuterated by growing the crystals from deuterated materials. In this work we have substituted As for $\mathrm{P}$, thereby obtaining mixed $\mathrm{Rb}_{1-x}\left(\mathrm{NH}_{4}\right)_{x} \mathrm{H}_{2} \mathrm{AsO}_{4}$ (RADA) crystals. While the spontaneous polarization in pure ferroelectrics of the KDP type has been studied, ${ }^{5-9}$ few experiments ${ }^{10-12}$ have been performed on spontaneous polarization in mixed crystals. Dielectric measurements ${ }^{13}$ in mixed RADA crystals for $0.04<x<0.10$ show coexistence of paraelectric/proton glass and ferroelectric order below the ferroelectric transition temperature. In this paper we extend previous results ${ }^{1+}$ on spon- 
taneous polarization of RADA and its deuterated counterpart and compare them to RDA and DRDA. Spontaneous polarization has also been calculated from the dielectric measurements along the $a$ axis of mixed crystals by a procedure to be explained, and compared to that obtained from saturated hysteresis loops.

Mixed crystals were obtained by slow evaporation of aqueous solutions of $\mathrm{RbH}_{2} \mathrm{AsO}_{4}$ (RDA) and $\left(\mathrm{NH}_{4}\right) \mathrm{H}_{2} \mathrm{AsO}_{4}$ (ADA) mixed in the proper molar ratios. The deuterated crystals were grown under an atmosphere of argon to maintain a high percentage of deuteration. The ferroelectric transition temperature $T_{\text {c }}$ depends on the percent of deuteration in the crystal. The $x$ values in the mixed crystals are determined from our measured $T_{c}$ 's with the aid of phase diagrams published for deuterated $^{15}$ and undeuterated ${ }^{16}$ RADA. Small platelets perpendicular to the $c$ tetragonal axis were cut from single crystals. Silver electrodes were evaporated in vacuum after polishing the surfaces to ensure that the faces were parallel. Polarization measurements were obtained from saturated dielectric hysteresis loops using the Sawyer-Tower circuit with a $60 \mathrm{~Hz}$ voltage source.

Figure 1 shows the spontaneous polarization of the undeuterated RADA sample. The ferroelectric transition for $x=0$ is first order ${ }^{y .17}$ and the transition temperature $T_{c}$ is found to be $110 \mathrm{~K}$. This is evidenced by the sharp rise in spontaneous polarization at $T_{c}$. The spontaneous polarization reaches a value of $(3.6 \pm 0.5) \mu \mathrm{C} \mathrm{cm}^{-2}$ at temperatures far below $T_{c}$. The large error bars in the spontaneous polarization results from the uncertainty in computing the surface area of the sample due to its small size. Within the limits of our experimental error this value agrees with that reported by Kamysheva et al. ${ }^{9}$ who have obtained a maximum value of $(4.2 \pm$ $0.2) \mu \mathrm{C} \mathrm{cm}^{-2}$ from specific heat measurements.

From the expression of the free energy $(G)$ as a function of polarization $(P)$ :

$$
G(P, T)=G_{0}(T)+\frac{\alpha}{2} P^{2}+\frac{\beta}{4} P^{4}+\frac{\gamma}{6} P^{n}+\ldots
$$

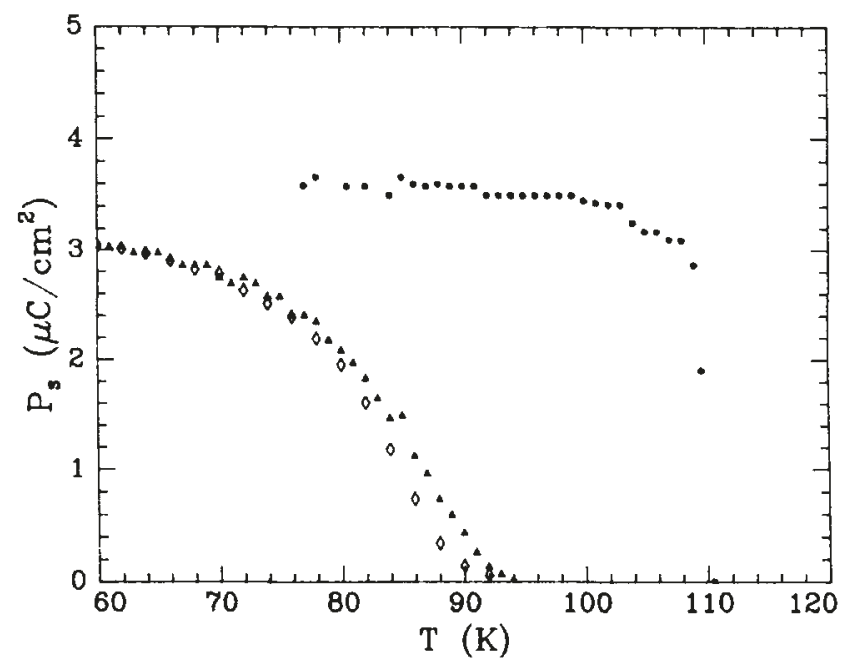

FIGURE 1 Spontaneous polarization obtained from saturated hysteresis loops in RDA $(x=0 ; \bullet)$ and RADA $(x=0.08 ; \Delta)$ as a function of temperature. The open diamond symbol represents spontaneous polarization obtained from Equation (3) and Figure 3 for RADA $x=0.08$. 
where $\alpha=\alpha_{0}\left(T-T_{0}\right)=4 \pi\left(T-T_{0}\right) / C, C$ is the Curie-Weiss constant and $T_{0}$ is the Curie-Weiss temperature, we have calculated the thermodynamic coefficients $\beta$ and $\gamma$ for the pure crystals. In Figure 2 we show the dielectric constant as a function of temperature measured along the $c$ axis at $1 \mathrm{kHz}$ and very low applied electric field $(10 \mathrm{~V} / \mathrm{cm})$ for the pure crystals. The solid line is a fit to the CurieWeiss law:

$$
\varepsilon_{c}^{\prime}-\varepsilon_{c x}=C /\left(T-T_{0}\right)
$$

This fit gives a value of $C=2200 \pm 20 \mathrm{~K}$ and $T_{0}=104 \mathrm{~K}$ for RDA; we have assumed a value of 10 for the "infinite frequency" dielectric response $\varepsilon_{c \%}$. Using this value for $C$ we calculate $\beta=-16.7 \times 10^{-10} \mathrm{c}$.g.s. and $\gamma=15.4 \times 10^{-1 \mathrm{~s}}$ c.g.s. from the expression $\left(\Delta P_{s}\right)^{2}=-3 \beta / 4 \gamma$ and $\left(\Delta P_{s}\right)^{2}=-4 \alpha / \beta$ where $\Delta P_{s}=$ $3.0 \mu \mathrm{C} \mathrm{cm}^{-2}$ is the jump in the spontaneous polarization at $T_{c}$. It must be pointed out that Equation (1) truncated at the $P^{6}$ term cannot be expected to fit such a big $P_{s}\left[\Delta P_{s} \approx P_{s}(0 \mathrm{~K})\right]$ very well. Table I shows the corresponding values obtained from saturated hysteresis loops ${ }^{8}$ and from specific heat measurements. ${ }^{9}$ The difference

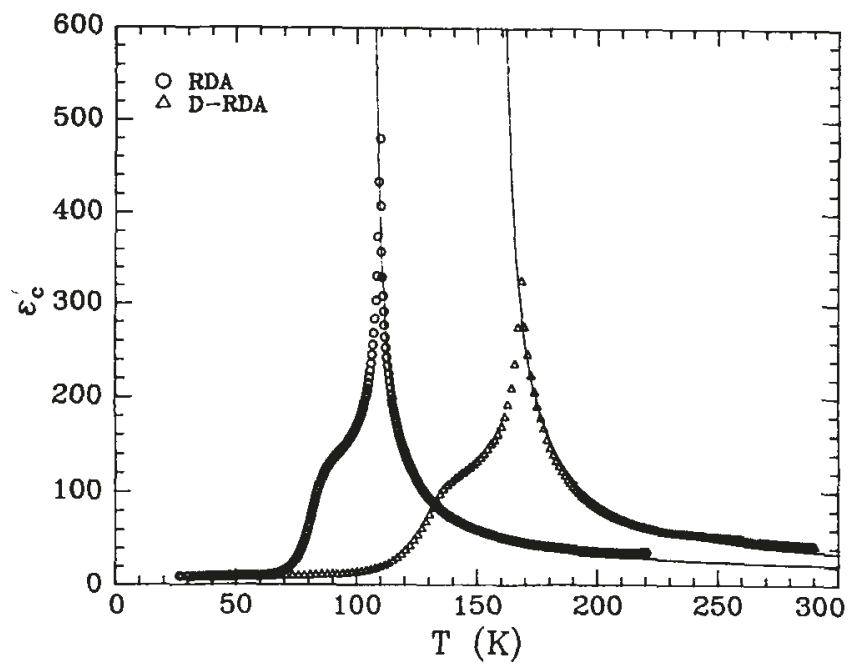

FIGURE 2 Real part of the dielectric permittivity $\varepsilon_{c}^{\prime}$ at $1 \mathrm{kHz}$ as a function of temperature along the $c$ axis, $(O)$ for RDA and $(\triangle)$ for DRDA. Solid line represents a fit to the Curie-Weiss law of Equation (2).

TABLE I

Tabulated values of $\beta$ and $\gamma$ defined in Equation (1) for RDA and DRDA together with fitting parameters defined in the text

\begin{tabular}{|c|c|c|c|c|c|c|}
\hline Ref. & Sample & $\mathrm{C}(\mathrm{K})$ & $T_{0}(\mathrm{~K})$ & $\begin{array}{c}\Delta \mathrm{P}_{\mathbf{s}^{-2}} \\
\mu \mathrm{Cm}^{-2}\end{array}$ & $\begin{array}{c}\mathrm{B} \\
\mathbf{c} \mathbf{g s}\end{array}$ & $\begin{array}{c}\gamma \\
\mathbf{c g s}\end{array}$ \\
\hline $\mathbf{8}$ & RDA & -- & 108.5 & 3.7 & $-20 \times 10^{-11}$ & $12 \times 10^{-19}$ \\
9 & RDA & 3100 & 108.5 & 3.6 & $-6.9 \times 10^{-11}$ & $4.4 \times 10^{-19}$ \\
This work & RDA & 2200 & 104 & 3.0 & $-16.7 \times 10^{-10}$ & $15.4 \times 10^{-18}$ \\
This work & DRDA & 3500 & 156 & 4.5 & $-9.4 \times 10^{-10}$ & $3.8 \times 10^{-18}$ \\
\hline
\end{tabular}


in our values arises from the low $T_{0}$ and $C$ values obtained from fitting Equation (2) to Figure 2.

The spontaneous polarization for $x=0.08$ RADA is also shown in Figure 1, and can be compared to that for the pure ferroelectric. The spontaneous polarization rises gradually in this case due to the presence of the ammonium cation centers, many of which are still in the paraelectric phase for some temperature range below $T_{c}$. However, at temperatures below $55 \mathrm{~K}$ the applied electric field needed to obtain saturated hysteresis loops exceeds the dielectric breakdown field of the sample, hence saturation polarization could not be computed below this temperature. The value of the spontaneous polarization measured at the lowest temperature reported is $(3.0 \pm 0.5) \mu \mathrm{C} \mathrm{cm}^{-2}$. We note that this value of the saturation polarization is less than that for the pure sample.

To explain this gradual polarization increase and lower maximum value, we assumed that the spontaneous polarization in the mixed crystal is equal to the spontaneous polarization in the pure ferroelectric well below $T_{c}$ multiplied by the fraction of the mixed crystal that goes ferroelectric below $T_{c}$. To determine this fraction, we performed dielectric measurements along the $a$ axis for the same mixed crystals. The glass transition as evidenced by dielectric dispersion is not seen in this sample at the temperatures covered in this experiment but is seen at lower temperatures. ${ }^{13}$ The spontaneous polarization predicted in this manner is given by:

$$
P_{s d}(T)=P_{s o}\left(\frac{\varepsilon_{1}^{\prime}(T)}{\varepsilon_{1}^{\prime}(T)+\varepsilon_{2}^{\prime}(T)}\right)
$$

where $P_{s d}$ is the spontaneous polarization obtained from the dielectric data, $P_{s o}$ is the maximum spontaneous polarization of the pure crystal well below $T_{c}$ and $\varepsilon_{1}^{\prime}$ and $\varepsilon_{2}^{\prime}$ are defined in Figure 3. Here, the "missing" dielectric response $\varepsilon_{1}^{\prime}$ is proportional to the ferroelectric portion and $\varepsilon_{2}^{\prime}$ represents the contribution to the permittivity from the paraelectric portion of the $x=0.08$ sample. The permittivity

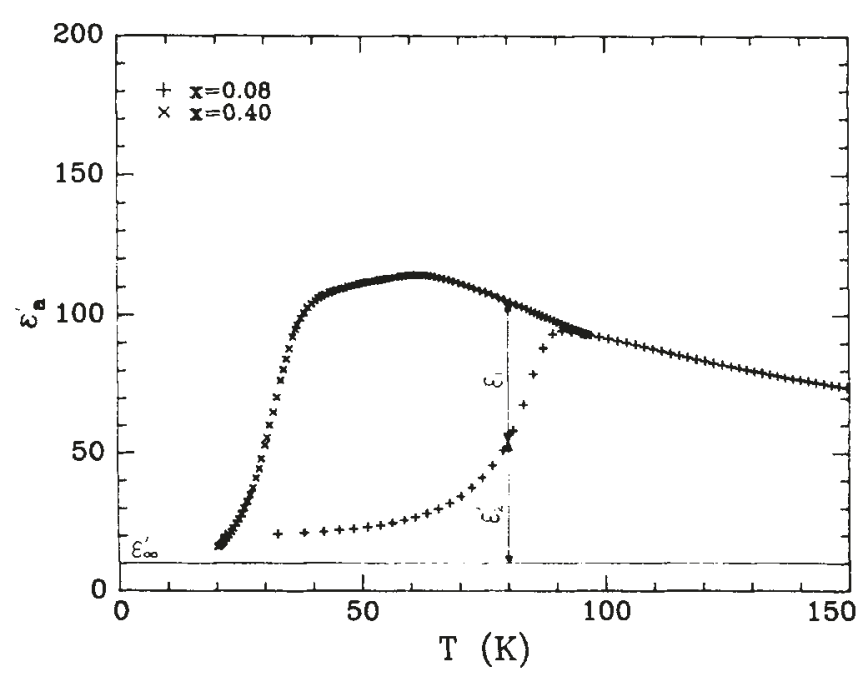

FIGURE 3 Real part of the dielectric permittivity $\varepsilon_{a}^{\prime}$ for the undeuterated mixed crystals at $1 \mathrm{kHz}$ as a function of temperature along the $a$ axis. $\varepsilon_{x}^{\prime}$ is assumed to be 10 . 
$\varepsilon_{1}^{\prime}+\varepsilon_{2}^{\prime}$ which represents the contribution from the same sample in the absence of the ferroelectric phase transition is estimated from dielectric data for RADA $x=$ 0.40 which has been included to help extrapolate qualitatively the Curie-Weiss behavior in the $x=0.08$ crystal to temperatures below $T_{c}$. The fraction ferroelectric below $T_{c}$ can be written as $\varepsilon_{1}^{\prime} /\left(\varepsilon_{1}^{\prime}+\varepsilon_{2}^{\prime}\right)$. Equation (3) is valid for temperatures above the nonergodic temperature and below $T_{c}$. In Figure 1 we show the variation in $P_{s d}$ as a function of temperature below $T_{c}$ and compare it to that obtained for the same sample from saturated hysteresis loops. The decrease in $P_{s d}$ to zero is sensitive to the way in which the two $\varepsilon_{a}^{\prime}(T)$ curves meet in Figure 3 . The agreement is seen to be good within the limits of experimental error. Here we have used $P_{s o}$ $=3.6 \mu \mathrm{C} \mathrm{cm}-2$ from Figure 1 .

In the case of mixed RADP samples, the spontaneous polarization again increases gradually but is found to approach the value of the pure ferroelectric RDP ${ }^{11}$ at low temperatures. This indicates coexistence of paraelectric and ferroelectric phases over a limited temperature range, below which the RADP crystals are completely ferroelectric.

Figure 4 shows the spontaneous polarization in the case of the deuterated samples. Here, deuteration effects raise the transition temperatures and the values of the spontaneous polarization as compared to the undeuterated counterpart. Similar effects of deuteration are seen in the phosphates. ${ }^{5.6}$ The transition in the pure sample is sharp when compared to the mixed sample. The value of the spontaneous polarization below $T_{c}$ for the pure sample is $(4.7 \pm 0.2) \mu \mathrm{C} \mathrm{cm}^{-2}$. The variation in the spontaneous polarization in the $x=0.08$ sample is gradual as in the case of the undeuterated sample and approaches a value of $(4.2 \pm 0.2) \mu \mathrm{C} \mathrm{cm}^{-2}$ at the lowest temperatures measured. From Figure 2, the Curie-Weiss fit for DRDA yields $C=3500 \pm 50 \mathrm{~K}$ and $T_{0}=156 \mathrm{~K}$. $T_{c}$ for DRDA is found to be $168 \mathrm{~K}$. This increase of about $1000 \mathrm{~K}$ for the Curie-Weiss constant in the deuterated sample is consistent with that observed in $\mathrm{KDP}^{6}$ and $\mathrm{RDP}^{7}$ upon deuteration. Using this

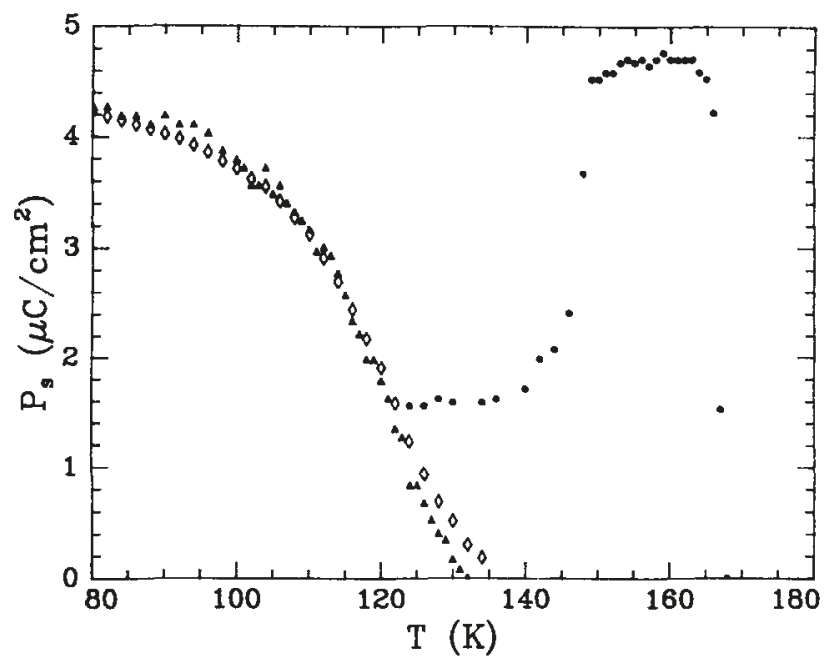

FIGURE 4 Spontaneous polarization obtained from saturated hysteresis loops in DRDA $(x=0 ; \bullet)$ and DRADA $(x=0.08 ; \Delta)$ as a function of temperature. The open diamond symbol represents spontaneous polarization obtained from Equation (3) and Figure 5 for DRADA $x=0.08$. 
value for $C$ we obtain for DRDA, $\beta=-9.4 \times 10^{-111}$ c.g.s. and $\gamma=3.8 \times 10^{-18}$ c.g.s. where we have used $\Delta P_{3}=4.5 \mu \mathrm{C} \mathrm{cm}^{-2}$ as the jump in the spontaneous polarization at $T_{c}$. These values are tabulated in Table I. We have calculated $P_{s d}$ for the deuterated case from Figure 5 and Equation (3) by a method analogous to that used to calculate the spontaneous polarization from the dielectric data in the undeuterated crystals. From Figure 4 we have used $P_{s o}=4.7 \mu \mathrm{C} \mathrm{cm}^{-2}$. In Figure 5 the glass transition for the $x=0.08$ crystal at $1 \mathrm{kHz}$ is seen around $43 \mathrm{~K}$ where the dielectric constant begins dropping faster with decreasing temperature. Here too, dielectric data for $x=0.28$ have been included to help extrapolate the CurieWeiss behavior of the $x=0.08$ crystal below $T_{\imath}$. The result is plotted in Figure 4 and can be compared to that obtained for the same sample from saturated hysteresis loops.

In Figure 4 we notice a drop in the spontaneous polarization for the pure sample below $153 \mathrm{~K}$. This effect was seen in two separate samples. Below $120 \mathrm{~K}$ the hysteresis loops could not be saturated because the necessary electric field would exceed the dielectric breakdown field of the crystal. However, saturated loops appeared above $120 \mathrm{~K}$ with increasing polarization as temperature was increased. This effect could indicate pinning of ferroelectric domains due to crystal defects or impurities at lower temperatures, thus giving a reduced value of spontaneous polarization. Further investigation of this effect is planned.

The experimental results show that deuteration increases the maximum value of the spontaneous polarization in the pure crystals. Also, the maximum value of the spontaneous polarization in the mixed crystals is lower than that of the pure crystal. This indicates that at the lowest temperatures recorded there are still paraelectric clusters intimately interlocked with the ferroelectric clusters. This type of coexistence is also seen ${ }^{1.3}$ down to $5 \mathrm{~K}$ from dielectric permittivity in these RADA mixed crystals. The gradual increase in spontaneous polarization below $T_{c}$ in the mixed crystals follows the gradual decrease in the dielectric constant of the corresponding crystals below $T_{\text {. }}$.

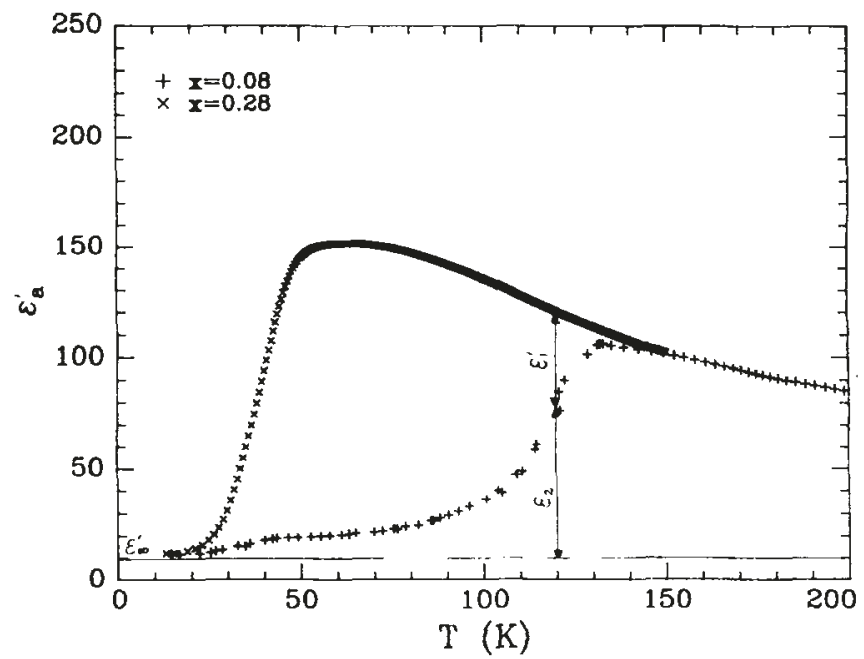

FIGURE 5 Real part of the dielectric permittivity $\varepsilon_{a}^{\prime}$ for the deuterated mixed crystals at $1 \mathrm{kHz}$ as a function of temperature along the $a$ axis. $\varepsilon_{x}^{\prime}$ is assumed to be 10 . 


\section{ACKNOWLEDGEMENTS}

The authors would like to thank Dr. S. Waplak for his encouragement in getting this project started and Dr. S. L. Hutton for automating the dielectric permittivity apparatus. This work was supported by National Science Foundation Grant DMR-9017429 and Department of Energy Equipment Grant DOE-FG05-91ER79046.

\section{REFERENCES}

1. E. Courtens, J. Phys, Letl., 43, L-199 (1982).

2. C. B. Sawyer and C. H. Tower, Phys. Rev., 35, 269 (1930).

3. A. Levstik, C. Filipič, Z. Kutnjak, I. Levstik, R. Pirc, B. Tadić and R. Blinc, Phys. Rev. Lett., 66, 2368 (1991).

4. N. J. Pinto, K. Ravindran, V. H. Schmidt and S. L. Hutton, Bull. Am. Phys. Soc., 37, 236 (1992).

5. G. A. Samara, Ferroelectrics, 5, 25 (1973).

6. B. A. Strukov, A. Baddur, V. N. Zinenko, A. V. Mishchenko and V. A. Koptsik, Sov. Phys. Solid State, 15, 939 (1973).

7. C. W. Fairall and W. Reese, Phys. Rev. B, 6, 193 (1972).

8. I. S. Zheludev, V. V. Gladkii, E. V. Sidnenko and V. K. Magataev, Ferroelectrics, 8, 567 (1974).

9. L. N. Kamysheva. Yu. S. Zolototrubov and S. A. Gridnev, Ferroelectrics, 8, 559 (1974).

10. S. A. Gridnev, L. N. Korotkov, L. A. Shuvalov and R. M. Fedosyuk, Sov. Phys. Solid State, 36, 522 (1991).

11. E. Courtens, Helv. Phys. Acta, 56, 705 (1983).

12. Y. Ono, T. Hikita and T. Ikeda. Ferroelectrics. 79, 327 (1988).

13. F. L. Howell, N. J. Pinto and V. H. Schmidt. Phys. Rev. B. 46, 13762 (1992).

14. N. J. Pinto, S. Waplak, S. L. Hutton. F. L. Howell and V. H. Schmidt, Bull. Am. Phys. Soc., 37, 287 (1992).

15. J. P. DeLooze, B. MacG. Campbell, N. S. Dalal and R. Blinc, Physica B, 162, 1 (1990).

16. Z. Trybula, V. H. Schmidt and J. E. Drumheller, Phys. Rev. B. 43, 1287 (1991).

17. R. Blinc, M. Burgar and A. Levstik, Solid State Commun., 12, 573 (1973). 